\title{
Age-Related Glucose Metabolism in FDG-PET Brain Studies
}

\section{Fatemeh Seifar', Farid Hajibonabi', Neda Parnianfard', Mahnaz Ranjkesh', Ebrahim Valikhani ${ }^{2}$ and Mohammad Yazdchi ${ }^{3^{*}}$}

\author{
${ }^{1}$ Student Research Committee, Tabriz University of Medical Sciences, Tabriz, Iran \\ ${ }^{2}$ Department of Radiology, Tabriz University of Medical Sciences, Tabriz, Iran \\ ${ }^{3}$ Neuroscience Research Center, Tabriz University of Medical Sciences, Tabriz, Iran
}

\begin{abstract}
In recent years, functional brain imaging modalities have been used as key tools for studying brain regional and global processes in healthy adults, as well as the pathophysiology and course of neurologic and psychiatric disorders. Positron emission tomography with Fluoro-deoxy glucose (FDG-PET) is widely used to measure regional cerebral glucose metabolic patterns. This review study focused on FDG PET findings in normal brain aging process, specifically on glucose metabolism in particular regions of brain. Based on our study, age-related reduction in glucose metabolism was significant in frontal, temporal and caudate part of basal ganglia, while the lowest changes found mostly in cerebellum, occipital and parietal regions. On the other hand, there was an increase in the glucose uptake by age in thalamic regions.
\end{abstract}

Keywords

Aging, Glucose uptake, Cerebral regions, Imaging, Positron emission tomography

\section{Aging in the Brain}

In recent years, functional brain imaging modalities have been used as key tools for studying brain regional and global processes in healthy adults, as well as the pathophysiology and course of neurologic and psychiatric disorders. Positron emission tomography with Fluoro-deoxy glucose (FDG-PET) is widely used to measure regional cerebral glucose metabolic patterns.

Aging in the brain entails biological, structural and chemical changes in brain. Studies have reported age-related loss in synaptic density, dendritic arborization and postsynaptic areas of the neocortical region and hippocampus. A hallmark of brain aging is a decrease of volume in the cortex, especially in Alzheimer disease patients [1]. Age-related neurodegenerative diseases such as Alzheimer's diseases are associated with the functional and structural changes. The decrease in cerebral glucose metabolism is known as the early event in the pathology of Alzheimer's diseases and may precede the neuropathological Amyloid beta $(A \beta)$ accumulation. The mechanism is related to the disturbed brain insulin metabolism by the alteration in insulin/insulin-like growth factor- 1 signaling in the affected patients [2,3].

Brain activity is also believed to decline during aging.
In the resting state, glucose regional cerebral metabolic rate (rCMRglc) is considered a determinant of brain functional activity. Glucose is oxidized in the neurons for ATP production to maintain normal function. Thus, brain glucose uptake is a parameter which is generally expected to reduce in the elderly [4].

PET is a conventional nuclear imaging modality, which allows conducting semi-quantitative investigations based on the body's uptake of a radioactive labeled molecule (in contrast to CMRglc which is quantitative). In brain studies this is often FDG, a radioactive labeled molecule, which is a marker of glucose metabolism. Here, we focused on FDG PET findings in normal brain aging process, specifically on glucose metabolism in particular regions of brain.

\begin{abstract}
${ }^{*}$ Corresponding author: Mohammad Yazdchi, Neuroscience Research Center, Imam Reza Hospital, Tabriz University of Medical Sciences, Golgasht Street, Tabriz, Iran, Tel: 00989141165992, E-mail: yazdchim@yahoo.com
\end{abstract}

Received: May 30, 2018; Accepted: June 12, 2018; Published online: June 14, 2018

Citation: Seifar F, Hajibonabi F, Parnianfard N, et al. (2018) Age-Related Glucose Metabolism in FDG-PET Brain Studies. Transl Neurosci Res Rev 1(1):5-9

Copyright: (c) 2018 Seifar F, et al. This is an open-access article distributed under the terms of the Creative Commons Attribution License, which permits unrestricted use, distribution, and reproduction in any medium, provided the original author and source are credited. 


\section{Regional Glucose Metabolism}

\section{Frontal lobe}

Frontal cortex consists of a variety of anatomical areas with heterogeneous and elusive tasks. It's divided in prefrontal cortex, dorsolateral and orbitofrontal regions. This region is most specifically associated with motor functions and organization aspects of memory.

De santa, et al. showed a $20-24 \%$ age-related reduction in glucose metabolism of frontal cortex, which was greater than temporal lobe changes. They also concluded that dorsolateral part -which is a core structure in working memory- was the main affected region with 2.6 moles $/ 100 \mathrm{~g} / \mathrm{min}$ decrease of glucose metabolism per age decade [5]. Loessner, et al. also found that glucose metabolism in frontal cortex was significantly decreased by age compared to other cerebral regions [6].

In recent studies, the detailed alteration in glucose metabolism of each region was evaluated. De Leon, et al. found left inferior frontal gyrus as the main area of the changes in aging [7]. This part also affected bilaterally in elder subjects.

Curati, et al. marked orbitofrontal cortex as a hypometabolic region of elder individuals' brains [8]. Inoue, et al., proposed that possible pathogenesis of reduction in frontal glucose metabolism in may correlate to disruption of synaptic activity of neural fibers. They found that neuronal functions of signaling in the frontal cortices decreased with the deterioration of the microstructures in corpus callosum without corresponding gray matter atrophy [9].

\section{Temporal lobe}

Neuropsychiatric researches have shown that aging is more related to changes in temporal lobe. The principal regions in this lobe are the medial temporal lobe and hippocampus, which are also involved in memory. These structures interfere with encoding and retention of new data and some aspects of retrieval. The MR imaging studies concentrated on the effect of aging on the volume and function of temporal lobe and specifically the medial part, which plays an essential role in cognition. De santi, et al. found that the glucose metabolism in temporal lobe was 13 to $23 \%$ lower in elder subjects compared to younger ones. They also reported that lateral temporal lobe was more affected by age compared to hippocampus ( $2.2 \mathrm{moles} / 100 \mathrm{~g} / \mathrm{min}$ decrease per decade compared to $1.4 \mathrm{moles} / 100 \mathrm{~g} / \mathrm{min}$ decrease) [5]. However other studies reported undifferentiated changes in hippocampus metabolism by age [6].

Temporo-parietal cortex has been regarded as age-dependent area of metabolism [7]. The most recent study by Bonte, et al. used PVE corrected 18F-FDG PET scans of elderlies for mapping the age dependent metabolism in brain regions, where a decreasing trend was reported in the temporal and frontal lobe [10].

Since the cognitive dysfunction is a main feature of aging, the temporal cortex and especially medial temporal lobe were considered as the core of glucose reduction in elderlies [11,12].

There is also evidence proving the pathological role of neuron loss and low glucose metabolism of hippocampus and medial temporal lobe in old patients with disabilities and dementia $[13,14]$.

\section{Parietal lobe}

The parietal lobe is part of a sensory system which works by integrating visual and somatosensory information. It is comprised of three major areas: Postcentral gyrus, also known as the sensory strip, supramarginal and angular gyrus which are involved in language [15]. The knowledge about brain parietal metabolic rates in aging is not well understood. Some studies in 80s confirmed a lower glucose uptake in PET images of the elderlies, while the localization of the hypometabolic patterns were not established [16-18]. Recent data also questions the age-related changes in parietal cortex. In volumetric studies of aging brains, parietal lobe atrophy was rare. These findings were also associated with little changes in metabolism with aging [10].

\section{Occipital lobe}

The occipital lobe is the visual processing center of the human brain. The primary visual cortex is in this area. The occipital region is known as a less affected area in aging $[19,20]$. Some studies also mentioned a significant increase in glucose metabolism in the occipital region $[21,22]$, which may be due to mechanism of compensation in the brain. The occipital lobe also remained unchanged by age both in size and metabolism based on Bonte, et al. study [10]. There is evidence that visual cortex also remains metabolically intact with advancing age $[6,22]$.

\section{Cerebellum}

The cerebellum consisted of two hemispheres, vermis and flocculonodular lobe which involved in coordination of voluntary movements such as coordination, gait, posture and speech. Though the cerebral association with age-related declines in cognitive performance and motor functions are well-understood, the possible contribution of the cerebellum is not. Based on literature examining the psychomotor performance of old and young subjects, there is a significant age-related decline. While some researchers showed modest changes in glucose uptake in 
the cerebellar region of old individuals [10,23], De santi, et al. found that cerebellum metabolism remains unchanged during normal aging process [5], which was also confirmed by Loesssner, et al. [6]. The data also support the hypothesis that cerebellum would differ in size by age but it would be relatively spared from this age-related decline in metabolism $[19,22]$. Therefore, the changes in the psychomotor function of older subjects may contribute, at least in part, to some of the age-dependent motor and cognitive performance declines in cerebral hemispheres.

\section{Thalamus}

The thalamus is a fundamental part in the coordination of data interring in the brain. It deals with information processing and component process of memory and executive functions of attention. Neuroimaging demonstrated age-dependent thalamic volume loss and structural changes, which may be associated with cognitive function contribute to age-related cognitive decline. However, the reports about the relationship between the age-related glucose metabolism and thalamus are discrepant in older subjects, sometimes conflicting. For example, one study indicated a reduced metabolism with age in the thalamus [24], while others reported an increased glucose uptake in thalamic core $[22,25]$.

One of the theories for the increased age-related metabolism in some brain regions was to compensate the performance of hypometabolic regions especially in memory function [26]. Hazlett, et al. also contributed this phenomenon to the different patterns of performance in youth compared to elderly [27]. Fujimoto and collogues showed that significant linear increases of metabolic values with age in the thalamus, is gender-dependent. It would appear in male in their twenties to forties but later in females' brain. They proposed that increased metabolism of glucose in thalamus of elderly can be due to age-related hypometabolism in the frontal lobe in individuals during their twenties to forties [21].

\section{Basal ganglia}

The basal ganglia and related nuclei includes different subcortical cell groups involved primarily in motor control, as well as other motor related functions such as executive functions, motor learning, behavior and emotions. The term basal ganglia refer to nuclei consisted of striatum or caudate-putamen and globus pallidus located in the brain hemispheres and structures embedded in the pons (pedunculopontine nucleus), diencephalon (subthalamic nucleus) and mesencephalon (substantia nigra) [28].

There are also age-related changes in subcortical structures such as a reduction volume of basal ganglia (BG) nuclei [29], and degradation of the nigrostriatal dopamine system [30].
In a study, among subcortical structures only the putamen and globus pallidus had significant declines in metabolism with age, while in total they were unaffected from age-related changes [22]. Moreover, the negative correlation between glucose metabolism and age in the caudate was observed in two other studies with relatively small changes $[12,25]$.

In most of the neuroimaging researches mentioned above, caudate was the most affected part of basal ganglia in aging process. The caudate nucleus is a small structure, which may also deteriorate in volume by age. Therefore, the reduced glucose metabolism with age in the caudate could be resulted by partial volume effects of PET related to the limited spatial resolution [12]. Yet, recent studies, demonstrated similar findings in the basal ganglia. Nugent, et al. found a higher glucose metabolism in the subcortical areas of putamen and caudate in younger brains. The effect of aging was also differential in the metabolism of acetoacetate [31]. Knopman, et al. demonstrated a significand volume loss in basal ganglia and a notable decline in FDG uptake in the putamen with age, while its potential relevance to cognitive aging wasn't clear [32].

Therefore, the only possible changes in basal ganglia is due to caudate nucleus, which can explain the age-related motor slowing in elderlies.

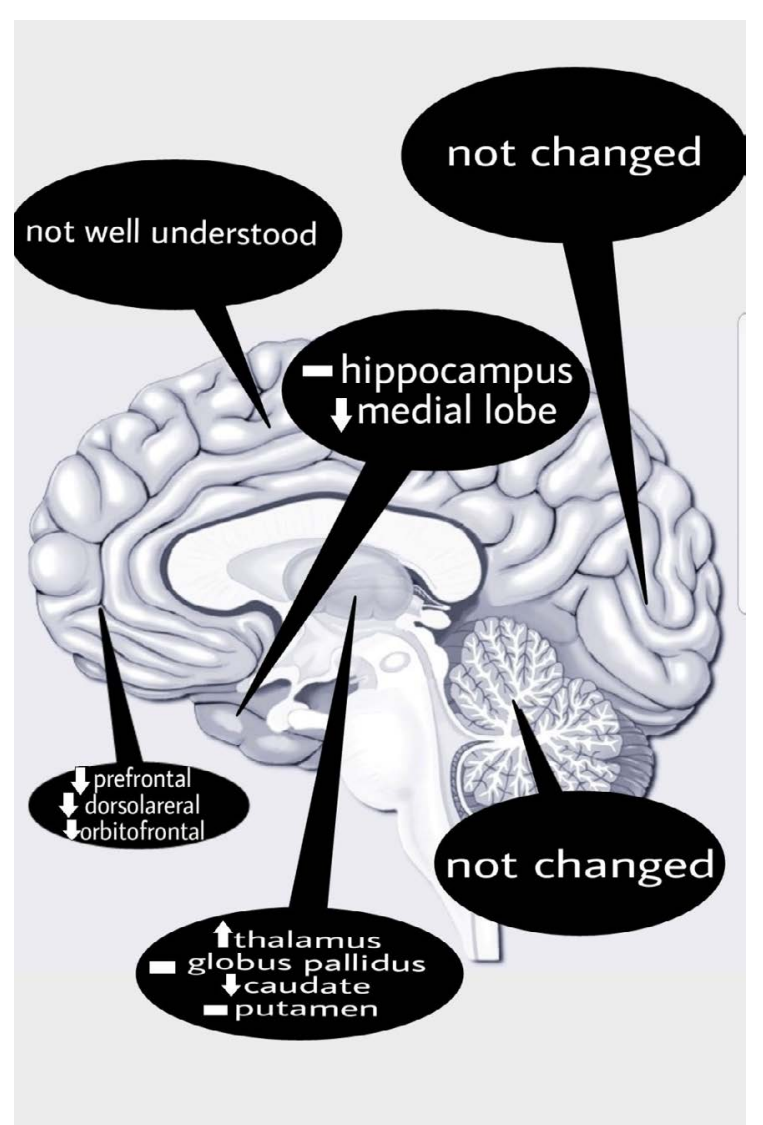

Figure 1: A summary of the effect of aging on brain glucose metabolism based on the studies. 
Citation: Seifar F, Hajibonabi F, Parnianfard N, et al. (2018) Age-Related Glucose Metabolism in FDG-PET Brain Studies. TransI Neurosci Res Rev 1(1):5-9

\section{Conclusion}

To sum up, different sample composition such as recruitment criteria and study population has led to some apparent variability in the results of different studies of age-related change in brain morphology and function. The studies mentioned earlier showed in healthy adults that even if brain atrophy is widespread, glucose metabolism varies across regions. On one hand significant age-related reduction in glucose metabolism was mostly present in frontal, temporal and caudate part of basal ganglia, while a lower decline was mostly in cerebellum, occipital and parietal regions. On the other hand, studies confirmed an increase in glucose uptake by age in thalamic regions. A brief review of the regional age-related changes in brain glucose metabolism is shown in Figure 1.

\section{References}

1. Creasey H, Rapoport SI (1985) The aging human brain Ann Neurol 17: 2-10.

2. Popa-Wagner A, Buga A-M, Popescu B, et al. (2015) Vascular cognitive impairment, dementia, aging and energy demand. A vicious cycle. J Neural Transm (Vienna) 122 S47-S54.

3. Freret T, Gaudreau P, Schumann-Bard P, et al. (2015) Mechanisms underlying the neuroprotective effect of brain reserve against late life depression. J Neural Transm (Vienna) 122: S55-S61.

4. De Keyser J, Ebinger G, Vauquelin G (1990) Age-related changes in the human nigrostriatal dopaminergic system. Ann Neurol 27: 157-161.

5. De Santi S, de Leon MJ, Convit A, et al. (1995) Age-related changes in brain: II. Positron emission tomography of frontal and temporal lobe glucose metabolism in normal subjects. Psychiatr Q 66: 357-370.

6. Loessner A, Alavi A, Lewandrowski KU, et al. (1995) Regional cerebral function determined by FDG-PET in healthy volunteers: Normal patterns and changes with age. J Nucl Med 36: 1141-1149.

7. de Leon MJ, Convit A, Wolf OT, et al. (2001) Prediction of cognitive decline in normal elderly subjects with $2-\left[{ }^{18} \mathrm{~F}\right]$ fluoro-2-deoxy-d-glucose/positron-emission tomography (FDG/PET). Proc Natl Acad Sci U S A 98: 10966-10971.

8. Curiati PK, Tamashiro-Duran JH, Duran FLS, et al. (2011) Age-related metabolic profiles in cognitively healthy elders: results from a voxel-based [18F]fluorodeoxyglucose-positron-emission tomography study with partial volume effects correction. AJNR Am J Neuroradiol 32: 560-565.

9. Inoue K, Ito H, Uchida S, et al. (2008) Decrease in glucose metabolism in frontal cortex associated with deterioration of microstructure of corpus callosum measured by diffusion tensor imaging in healthy elderly. Hum Brain Mapp 29: 375384.

10. Bonte S, Vandemaele P, Verleden S, et al. (2017) Healthy brain ageing assessed with 18F-FDG PET and age-dependent recovery factors after partial volume effect correction. Eur J Nucl Med Mol Imaging 44: 838-849.
11. Eberling JL, Nordahl TE, Kusubov N, et al. (1995) Reduced temporal lobe glucose metabolism in aging. J Neuroimaging 5: 178-182.

12. Shen X, Liu H, Hu Z, et al. (2012) The relationship between cerebral glucose metabolism and age: Report of a large brain PET data set. PLoS One 7: e51517.

13. Cao Q, Jiang K, Liu Y, et al. (2002) [The comparison of the regional cerebral metabolism rate of glucose in Alzheimer's disease with mild cognitive impairment]. Zhonghua Yi Xue Za Zhi 82: 1613-1616.

14. Allen JS, Bruss J, Brown CK, et al. (2005) Normal neuroanatomical variation due to age: The major lobes and a parcellation of the temporal region. Neurobiol Aging 26: 1245-1282.

15. Rushworth MF, Krams M, Passingham RE (2001) The attentional role of the left parietal cortex: The distinct lateralization and localization of motor attention in the human brain. J Cogn Neurosci 13: 698-710.

16. Alavi A, Dann R, Chawluk J, et al. (1986) Positron emission tomography imaging of regional cerebral glucose metabolism. Semin Nucl Med 16: 2-34.

17. Garraux G, Salmon E, Degueldre C, et al. (1999) Comparison of impaired subcortico-frontal metabolic networks in normal aging, subcortico-frontal dementia, and cortical frontal dementia. Neuroimage 10: 149-162.

18. Burns A, Tyrrell P (1992) Association of age with regional cerebral oxygen utilization: A positron emission tomography study. Age Ageing 21: 316-320.

19. Petit-Taboue MC, Landeau B, Desson JF, et al. (1998) Effects of healthy aging on the regional cerebral metabolic rate of glucose assessed with statistical parametric mapping. Neuroimage 7: 176-184.

20. Lee JS, Lee DS, Park KS, et al. (2004) Changes in the heterogeneity of cerebral glucose metabolism with healthy aging: Quantitative assessment by fractal analysis. J Neuroimaging $14: 350-356$.

21. Fujimoto T, Takeuch K, Matsumoto T, et al. (2007) Abnormal glucose metabolism in the anterior cingulate cortex in patients with schizophrenia. Psychiatry Res 154: 49-58.

22. Willis MW, Ketter TA, Kimbrell TA, et al. (2002) Age, sex and laterality effects on cerebral glucose metabolism in healthy adults. Psychiatry Res 114: 23-37.

23. Kalpouzos G, Chetelat G, Baron J-C, et al. (2009) Voxel-based mapping of brain gray matter volume and glucose metabolism profiles in normal aging. Neurobiol Aging 30: 112-124.

24. Pardo JV, Lee JT, Sheikh SA, et al. (2007) Where the brain grows old: Decline in anterior cingulate and medial prefrontal function with normal aging. Neuroimage 35: 1231-1237.

25. Kim I-J, Kim S-J, Kim Y-K (2009) Age- and sex-associated changes in cerebral glucose metabolism in normal healthy subjects: Statistical parametric mapping analysis of F-18 fluorodeoxyglucose brain positron emission tomography. Acta Radiol 50: 1169-1174.

26. Scarmeas N, Zarahn E, Anderson KE, et al. (2003) Cognitive reserve modulates functional brain responses during memory tasks: A PET study in healthy young and elderly subjects. Neuroimage 19: 1215-1227. 
Citation: Seifar F, Hajibonabi F, Parnianfard N, et al. (2018) Age-Related Glucose Metabolism in FDG-PET Brain Studies. TransI Neurosci Res Rev 1(1):5-9

27. Hazlett EA, Buchsbaum MS, Mohs RC, et al. (1998) Age-related shift in brain region activity during successful memory performance. Neurobiol Aging 19: 437-445.

28. Lanciego JL, Luquin N, Obeso JA (2012) Functional neuroanatomy of the basal ganglia. Cold Spring Harb Perspect Med 2: 009621.

29. Raz N, Rodrigue KM, Kennedy KM, et al. (2003) Differential aging of the human striatum: Longitudinal evidence. AJNR Am J Neuroradiol 24: 1849-1856.
30. Erixon-Lindroth N, Farde L, Wahlin T-BR, et al. (2005) The role of the striatal dopamine transporter in cognitive aging. Psychiatry Res 138: 1-12.

31. Nugent S, Tremblay S, Chen KW, et al. (2014) Brain glucose and acetoacetate metabolism: A comparison of young and older adults. Neurobiol Aging 35: 1386-1395.

32. Knopman DS, Jack CRJ, Wiste HJ, et al. (2014) 18F-fluorodeoxyglucose positron emission tomography, aging, and apolipoprotein $\mathrm{E}$ genotype in cognitively normal persons. Neurobiol Aging 35: 2096-2106. 